\title{
Synthesis of the Nakanishi Ring-Locked Retinoid
}

\author{
Jamie B. Côté, ${ }^{1}$ Tan D. Quach, ${ }^{1}$ Andrey P. Demenev, ${ }^{2}$ David S. Garvey, ${ }^{3}$ and Judd M. Berman ${ }^{1}$ \\ ${ }^{1}$ Dalton Medicinal Chemistry Inc., 349 Wildcat Road, Toronto, ON, Canada M3J 2S3 \\ ${ }^{2}$ Dalton Pharma Services, 349 Wildcat Road, Toronto, ON, Canada M3J 2S3 \\ ${ }^{3}$ Bikam Pharmaceuticals Inc., c/o Fidelity Biosciences, 1 Main Street, 13th Floor, Cambridge, MA 02142, USA \\ Correspondence should be addressed to Judd M. Berman, jberman@dalton.com \\ Received 15 June 2011; Accepted 18 July 2011 \\ Academic Editor: Armando Rossello \\ Copyright () 2011 Jamie B. Côté et al. This is an open access article distributed under the Creative Commons Attribution License, \\ which permits unrestricted use, distribution, and reproduction in any medium, provided the original work is properly cited.
}

\begin{abstract}
An optimized synthetic route to prepare ring-locked retinoid 1a has been developed. We fully describe a purification protocol that provides isomerically pure 1a in support of on-going proof of concept studies for the development of therapeutic agents to treat human ADRP. Additionally, we have found that isomerically pure 1a can be stored in amber vials under argon at $-20^{\circ} \mathrm{C}$ for use over time (up to six months) without degradation. Thus, enabling la to be an accessible and valuable biological tool.
\end{abstract}

Previous studies using $\mathrm{P} 23 \mathrm{H}$ mutant opsin as an in vitro model of human Autosomal Dominant Retinitis Pigmentosa (ADRP) found that ring-locked retinoid 1a (Figure 1, carbon numbering shown based on 11-cis-retinal) was able to act as a pharmacological chaperone [1-3]. Compound 1a induced the mutant protein to fold properly and undergo normal cellular transport and degradation suggesting 1a could be a potential therapeutic agent for the prevention of ADRP [1-3]. This ring-locked analog of 11-cis-retinal has also been used extensively to elucidate the mechanism of photoactivation of rhodopsin [4-6].

We required a reliable synthesis of isomerically pure compound 1a. Its synthesis, along with the spectral data, has been reported twice in the literature $[1,7]$, in addition to the syntheses of a number of related ring-locked retinoids $[4-6,8]$. The original synthetic route reported by Nakanishi resulted in the preparation of mixtures of multiple geometric isomers of 1 (E/Z-isomers among C7-C8, C9-C10, and C13-C14) and did not describe specific HPLC conditions for resolution of the final isomerically pure products. Our synthetic strategy differs from the two reported syntheses in two fundamental ways (discussed below) enabling ready access to 1a. Two key bond-forming events distinguish this synthesis: the C7-C8 double bond was originally established via a Julia olefination, while the connection between C13 and $\mathrm{C} 14$ was accomplished via a Petersen olefination. In each case, both $E$ - and $Z$-isomers of the desired products were reported to have formed [7]. In contrast, our use of a Wittig reagent in the formation of the C7-C8 double bond afforded the E-isomer as the sole product in the former olefination; and in the case of the latter, the requisite Petersen reagent, a silylated acetaldehyde tert-butylamine, was not readily accessible in our hands, and an alternate methodology was instead utilized $[7,9]$. A Horner-EmmonsWadsworth strategy (utilizing diethyl cyanomethylphosphonate) was found to be an advantageous replacement for the penultimate formation of the C13-C14 double bond in two steps (as opposed to three as previously reported) [1]. In particular, using the nitrile Horner-Emmons-Wadsworth (Scheme 2) reagent obviated the need for a final manganese dioxide oxidation which resulted in a more efficient and reliable synthesis endgame for the preparation of $\mathbf{1 a}$. The key changes we have introduced to the synthetic route as described by Noorwez et al. [1] allowed us to increase the yields and geometric purity of key intermediates ( 7 , 11). Herein, we report the full details of the synthesis, purification, characterization, and stability of isomerically pure 1a.

Starting from commercially available cycloheptenone 2, the allylic acetate 4 was prepared via a two-step sequence of radical bromination with NBS and $1,1^{\prime}$ azobis(cyclohexanecarbonitrile) (ACHN) followed by displacement of the allylic bromide 3 with potassium acetate under phase transfer catalyst conditions with tetrabutylammonium bromide (TBAB, Scheme 1). The ketone underwent 


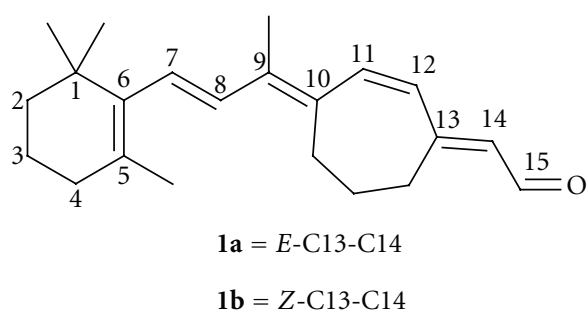

FIgURE 1: Ring-locked retinoid derivative of 11-cis-retinal.

Horner-Emmons-Wadsworth olefination with diethyl(1cyanoethyl)phosphonate to provide a 50\% yield of $\mathbf{5}$ and $\mathbf{6}$ with an $E: Z$ ratio of $2: 1$. The isomers were separated by column chromatography, and the geometry of the double bond was established by NOE (Figure 2). The allylic acetate 5 was hydrolyzed with potassium carbonate in methanol to furnish the allylic alcohol 7 in excellent yield and purity. The alcohol was protected as the TBS ether $\mathbf{8}$ in the presence of imidazole and DMAP, and then the nitrile was reduced with DIBAL-H to provide aldehyde 9 in $77 \%$ yield over two steps. The aldehyde 9 was carried forward without purification.

Importantly, the double-bond geometry (between C9 and C10) remained fixed during this three-step reaction sequence. The trans geometry was confirmed by NOE experiments (key interactions are shown in Figure 2).

The Wittig olefination reagent, $\beta$-cyclogeranyl triphenylphosphonium bromide, was prepared following established methodology $[10,11]$. Reaction conditions using $n$ $\mathrm{BuLi}$ as the base resulted only in decomposition. However, the desired tetraene $\mathbf{1 1}$ was obtained when potassium tertbutoxide was employed as the base in the presence of a catalytic amount of 18-crown-6 [12]. In addition, the newly established C7-C8 double bond was solely of the trans geometry. The cis isomer of $\mathbf{1 1}$ was not obtained or detected due to judicious choice of conditions, optimization of the reaction time/temperature, and the inherent instability of the $\mathrm{C} 7 / \mathrm{C} 8$ cis isomer due to the unfavorable sterics between the C5/C9 methyl groups. Interestingly, compound $\mathbf{1 1}$ was found to be quite stable when stored at $4^{\circ} \mathrm{C}$ in the dark under argon (no decomposition or isomerization detected after 18 months). The TBS protecting group was cleaved using TBAF to provide the corresponding allylic alcohol. In contrast to 11, we found the allylic alcohol readily decomposed under normal atmospheric conditions; thus, it was more convenient to oxidize it directly using $\mathrm{MnO}_{2}$ to provide ketone 12 which, in our hands, was more stable to manipulation. The Horner-Emmons-Wadsworth reaction on 12 with diethyl cyanomethylphosphonate provided the corresponding nitrile in excellent yield as a 1:1 mixture of $E$ and $Z$-isomers which, as reported on similar analogues, were not separable by HPLC [8]. Thus, reduction of the nitrile was achieved by treatment with DIBAL-H to the intermediate imine, and silica gel-mediated hydrolysis furnished the aldehydes $\mathbf{1 a}$ and $\mathbf{1} \mathbf{b}$ in high yield. The observed ratio of C13-C14 double-bond isomers and reaction yield is related to the conditions of the final step of synthesis. Hence, if hydrolysis of the intermediate imine from the conversion of 12 to 1 on silica gel is left longer than 30 minutes at room temperature, more isomerization and decomposition takes place (resulting in the appearance of three other peaks in the HPLC trace), and the yield of $\mathbf{1} \mathbf{a} / \mathbf{b}$ decreases. Under the conditions reported herein, the combined percentage of the other impurities in addition to the C13-C14 E/Z-isomers was minimal ( $4 \%$ AUC at $350 \mathrm{~nm}$ ). Both the C13-C14 E/Zisomers of $\mathbf{1}$ were readily separated by normal phase HPLC in a dark room (see Experimental). The isomers were identified by comparison of key chemical shifts in the ${ }^{1} \mathrm{H}$ NMR spectra to literature data (Table 1) $[1,7]$.

It was observed that the isomerization of the C13C14 double bond of 1 occurs readily in solution under normal atmospheric conditions regardless of exposure to light. Hence, a crude sample isolated from the reaction as a $1: 1$ mixture of $\mathbf{1 a}$ and $\mathbf{1} \mathbf{b}$ would isomerize between the two compounds.

To probe the isomers' stability to light and storage conditions, $2 \mathrm{mg}$ of each purified isomer was dissolved in hexane $(2 \mathrm{~mL})$ in a clear glass vial. Half of this solution was transferred into an amber glass vial. Each of the vials was flushed with argon and left to stand at room temperature in ambient light. After one hour, aliquots were removed from each vial and analyzed by HPLC. The samples in the amber vials remained unchanged, whereas the samples in the clear vials had isomerized to $13 \%$ (AUC at $350 \mathrm{~nm}$ ). The vials were flushed with argon again and left overnight at room temperature. Again, aliquots from each vial were analyzed by HPLC, the samples in the amber vials remained unchanged, but the samples in the clear vials had isomerized further to $31 \%$ (Figure 3).

Importantly, in a separate experiment, a purified sample of $1 \mathbf{a}$ was stored neat at $-20^{\circ} \mathrm{C}$ in an amber vial flushed with argon. It was found that the sample remained unchanged from isomerization or decomposition (via HPLC monitoring) after six months.

In conclusion, we have developed a synthetic route to prepare the ring-locked retinoid $\mathbf{1 a}$ and described a reproducible purification protocol by normal phase HPLC to isolate the isomerically pure C13-C14 Z-isomer of $\mathbf{1}$. Finally, we have found that under proper conditions $\mathbf{1}$ can be stored for extended periods of time (up to six months) without degradation.

${ }^{1} \mathrm{H},{ }^{13} \mathrm{C}$ spectra were recorded on a Bruker ARX 300, 400, or $600 \mathrm{MHz}$ spectrometers. Low-resolution mass spectra (MS) were obtained on a Thermo Finnigan LCQ Classic or LCQ Duo spectrometer. HPLC purifications were conducted on a Waters Prep LC 4000 equipped with a Waters plus 717 autosampler and a Waters 2487 dual $\lambda$ Absorbance Detector.

All commercial reagents were used as received, unless otherwise noted. All reactions, work-up, and purifications for compounds 1, 9-12 were performed in a dark room utilizing a red spectrum light source. Final compounds and isolated key intermediates were stored in amber glass vials at $-20^{\circ} \mathrm{C}$ under argon. Analytical thin-layer chromatography (TLC) was performed on glass-backed TLC plates (silica gel $60 \mathrm{~F}_{254}$ from Silicycle), visualized with a UV lamp $(254 \mathrm{~nm})$, and stained with a basic solution of $\mathrm{KMnO}_{4}$. Flash column chromatography was conducted on an ISCO 


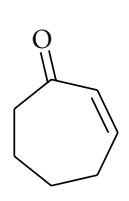

2

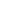

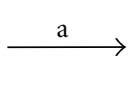<smiles>[R]C1C=CC(=O)CCC1</smiles>

$\mathrm{R}=\mathrm{Br}, \mathbf{3}$ $=\mathrm{OAc}, 4$ $\mathrm{b} \longrightarrow$<smiles>CC(=O)OC1C=CC(=C(C)C#N)CCC1</smiles>

$E: Z$

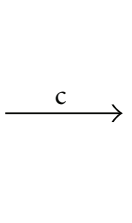<smiles>CC(=O)OC1C=CC(=C(C)C#N)CCC1</smiles>

$E, 5$<smiles>C[AlH]</smiles>

$\mathrm{OHC}$<smiles>CC(C)=C1C=CC([Hg]O)CCC1</smiles>

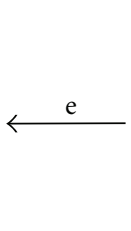

(1)

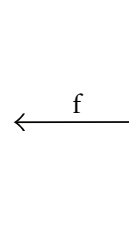<smiles>CC(C#N)=C1C=CC(O[Sb])CCC1</smiles>

8

9

Scheme 1: Reagents and conditions: (a) (i) NBS, ACHN, benzene, reflux, 2 h, and 34\%; (ii) KOAc, TBAB, THF/ $\mathrm{H}_{2} \mathrm{O}$, r.t., 4 days, and 17\%; (b) $\mathrm{NaH},(\mathrm{EtO})_{2} \mathrm{P}(\mathrm{O}) \mathrm{CH}\left(\mathrm{CH}_{3}\right) \mathrm{CN}$, THF, $0^{\circ} \mathrm{C}$ to r.t., and $20 \mathrm{~h}$; (c) isomer separation by $\mathrm{SiO}_{2}$ column chromatography, $E 33 \%$, and $Z 17 \%$; (d) $\mathrm{K}_{2} \mathrm{CO}_{3}, \mathrm{MeOH}$, r.t., $1.5 \mathrm{~h}$, and 99\%; (e) TBSCl, imidazole, $\mathrm{DMAP}, \mathrm{CH}_{2} \mathrm{Cl}_{2}$, r.t., $1 \mathrm{~h}$, and $80 \%$; (f) $\mathrm{DIBAL}-\mathrm{H}, \mathrm{Et}_{2} \mathrm{O},-78^{\circ} \mathrm{C}$ to $0^{\circ} \mathrm{C}, 2 \mathrm{~h}$, and $96 \%$.<smiles>CC(C=O)=C1C=CC(O[18F])CCC1</smiles>

9<smiles>CCCCC1=C(C)CCCC1(C)C</smiles>

10<smiles>CC1=C(/C=C/C(C)=C2/C=CC(=O)CCC2)C(C)(C)CCC1</smiles>

12

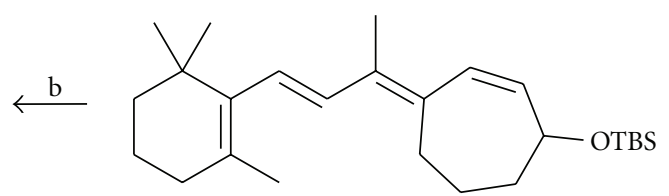

11

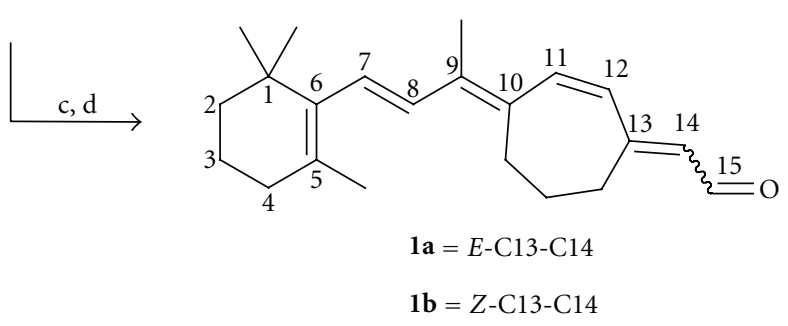

Scheme 2: Reagents and conditions: (a) $\mathrm{KO} t \mathrm{Bu}, 18$-crown-6, $\mathrm{CH}_{2} \mathrm{Cl}_{2}$, r.t., 5 h, and $35 \%$; (b) (i) TBAF, $\mathrm{THF}, 0^{\circ} \mathrm{C}$, and $4.5 \mathrm{~h}$; (ii) $\mathrm{MnO}_{2}, \mathrm{CH}_{2} \mathrm{Cl}_{2}$, $-20^{\circ} \mathrm{C}$ to $0^{\circ} \mathrm{C}, 2 \mathrm{~h}$, and $47 \%$; (c) $\mathrm{NaH}$, (EtO) ${ }_{2} \mathrm{P}(\mathrm{O}) \mathrm{CH}_{2} \mathrm{CN}$, THF, r.t., 3 h, and 99\%; (d) (i) DIBAL-H, $\mathrm{Et}_{2} \mathrm{O},-78^{\circ} \mathrm{C}$ to r.t., and 30 min; (ii) $\mathrm{SiO}_{2}$ gel, ether, and $\mathrm{E} / \mathrm{Z} 96 \%$.

Companion automated chromatographer using generic silica gel cartridges from Silicycle.

4-Oxo-cyclohept-2-en-1-yl Acetate (4) (See [12]). A solution of cycloheptenone $(2,80 \%$ tech. grade, $8.00 \mathrm{~g}, 73.0 \mathrm{mmol})$,
NBS (17.5 g, $98.0 \mathrm{mmol})$, and ACHN (180 mg, $0.730 \mathrm{mmol}$ ) in benzene $(150 \mathrm{~mL})$ was combined at r.t. under argon then heated at reflux for $3 \mathrm{~h}$. The reaction mixture was cooled to room temperature, then cooled to $0^{\circ} \mathrm{C}$ in an ice bath, and diluted with hexanes $(200 \mathrm{~mL})$. The succinimide 


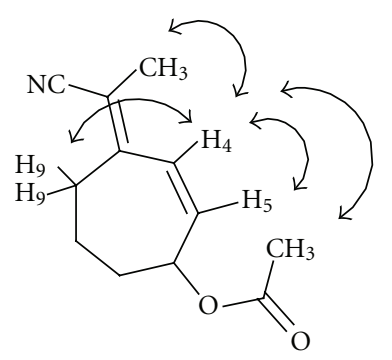

5

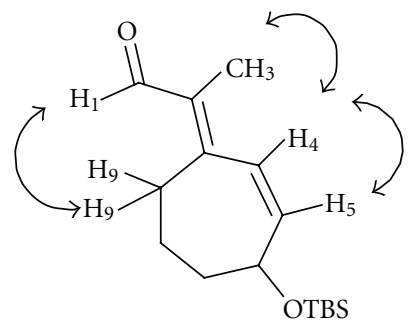

9

Figure 2: Key NOEs used to establish the geometry of the tetra-substituted double bond corresponding to the double bond between C9 and C10 of 1.

TABLE 1: Summary of the literature $[1,7]$ and experimental (exp) ${ }^{1} \mathrm{H}$ NMR chemical shifts of key resonances $(\delta$ reported in $\mathrm{ppm})$ for C13-C14 E and Z-isomers of 1.

\begin{tabular}{lcccccc}
\hline Proton & \multicolumn{3}{c}{$\mathrm{C} 13-\mathrm{C} 14$-isomer } & \multicolumn{3}{c}{$\mathrm{C} 13-\mathrm{C} 14$ E-isomer } \\
Reference & {$[1]$} & {$[7]$} & $\exp$ & {$[1]$} & {$[7]$} & $\exp$ \\
Solvent & $\mathrm{CDCl}_{3}$ & $\mathrm{CDCl}_{3}$ & $\mathrm{CD}_{2} \mathrm{Cl}_{2}$ & $\mathrm{CDCl}_{3}$ & $\mathrm{CDCl}_{3}$ & $\mathrm{CDCl}_{3}$ \\
\hline $\mathrm{H}-7$ & 6.32 & - & 6.40 & 6.32 & - & 6.38 \\
H-8 & 6.52 & - & 6.61 & 6.52 & - & 6.57 \\
H-11 & 7.02 & 7.00 & 7.07 & 6.92 & - & 6.95 \\
H-12 & & & & 6.22 & 6.21 & 6.26 \\
H-14 & 5.79 & 5.78 & 5.80 & 5.93 & 5.93 & 5.97 \\
H-15 & 10.12 & 10.11 & 10.15 & 10.03 & 10.03 & 10.07 \\
\hline
\end{tabular}

precipitate was removed by vacuum filtration, and the filtrate was concentrated in vacuo to provide 3 as a dark brown oil. The crude bromide 3 was dissolved in THF $(75 \mathrm{~mL})$ at room temperature. To this solution was added water $(50 \mathrm{~mL})$, potassium acetate $(28.0 \mathrm{~g}, 285 \mathrm{mmol})$, and tetrabutylammonium bromide $(230 \mathrm{mg}, 0.730 \mathrm{mmol})$. The biphasic mixture was stirred vigorously at r.t. for 4 days under argon. The reaction mixture was then transferred to a separatory funnel and extracted with ether $(3 \times$ $100 \mathrm{~mL}$ ). The combined organic phases were washed with saturated $\mathrm{NaHCO}_{3}(2 \times 150 \mathrm{~mL})$, brine $(150 \mathrm{~mL})$, dried over $\mathrm{MgSO}_{4}$, filtered, and concentrated in vacuo. The product was purified by column chromatography $(330 \mathrm{~g}$ silica gel cartridge, gradient elution 0-20\% EtOAc-hexanes), yield of

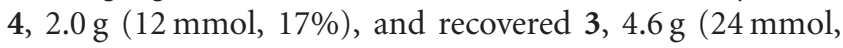
$34 \%)$.

$$
\left.R_{f}=0.21 \text { (EtOAc-hexanes, } 1: 8\right) .
$$

${ }^{1} \mathrm{H} \mathrm{NMR}\left(600 \mathrm{MHz}, \mathrm{CDCl}_{3}\right): \delta=6.42(\mathrm{dd}, 1 \mathrm{H}, J=12.5$, $3.5 \mathrm{~Hz}, \mathrm{H}-2), 6.00(\mathrm{dd}, 1 \mathrm{H}, J=12.5,1.5 \mathrm{~Hz}, \mathrm{H}-3), 5.56(\mathrm{~m}$, $1 \mathrm{H}, \mathrm{H}-1), 2.66-2.56$ ( $\mathrm{m}, 2 \mathrm{H}, \mathrm{H}-5), 2.22-2.15$ (m, $1 \mathrm{H}, \mathrm{H}-7$ ), 2.10-2.06 (s, 3H, $\left.\mathrm{O}_{2} \mathrm{CCH}_{3}\right), 1.90-1.81$ (m, 3H, H-6, H-7).

${ }^{13} \mathrm{C}$ NMR $\left(150 \mathrm{MHz}, \mathrm{CDCl}_{3}\right): \delta=18.0$ (C6), 21.0 $\left(\mathrm{O}_{2} \mathrm{CCH}_{3}\right), 31.6(\mathrm{C} 7), 42.8(\mathrm{C} 5), 71.9(\mathrm{C} 1), 131.3(\mathrm{C} 3), 144.3$ (C2), $169.9\left(\mathrm{O}_{2} \mathrm{CCH}_{3}\right), 202.3(\mathrm{C} 4)$.
4-Bromocyclohept-2-enone (3) (See [13]).

$R_{f}=0.34$ (EtOAc-hexanes, $1: 8$ ).

${ }^{1} \mathrm{H}$ NMR $\left(600 \mathrm{MHz}, \mathrm{CDCl}_{3}\right): \delta=6.50(\mathrm{dd}, 1 \mathrm{H}, J=12.5$, $5.5 \mathrm{~Hz}, \mathrm{H}-3), 5.90(\mathrm{~d}, 1 \mathrm{H}, J=12.5 \mathrm{~Hz}, \mathrm{H}-2), 4.99$ (dd, $1 \mathrm{H}, J=$ $10.0,5.5 \mathrm{~Hz}, \mathrm{H}-4), 2.81$ (ddd, $1 \mathrm{H}, J=17.0,7.5,4.5 \mathrm{~Hz}, \mathrm{H}-7$ ), 2.68-2.58 (m, 1H, H-7), 2.46-2.28 (m, 2H, H-5), 2.19-2.08 (m, 1H, H-6), 1.91-1.78 (m, 1H, H-6).

${ }^{13} \mathrm{C}$ NMR $\left(150 \mathrm{MHz}, \mathrm{CDCl}_{3}\right): \delta=19.2(\mathrm{C} 6), 36.4$ (C5), 43.8 (C7), 49.9, (C4), 130.6 (C2), 141.3 (C3), 202.3 (C1).

$$
\text { MS (APCI): } m / z[\mathrm{M}+\mathrm{H}]^{+}=189.1,191.1 \text {. }
$$

(E)-4-(1-Cyanoethylidene)cyclohept-2-en-1-yl Acetate (5) (See [12]). Into a dried flask fitted with an addition funnel was placed $\mathrm{NaH}$ ( $60 \%$ dispersion in oil, $200 \mathrm{mg}, 84.0 \mathrm{mmol}$ ) and THF $(140 \mathrm{~mL})$. The slurry was cooled in an ice bath, and the addition funnel was charged with a solution of diethyl(1cyanoethyl)phosphonate $(1.06 \mathrm{~mL}, 6.00 \mathrm{mmol})$ in THF $(45 \mathrm{~mL})$. The phosphonate solution was added slowly over 5 minutes to the $\mathrm{NaH}$ slurry at $0^{\circ} \mathrm{C}$. The reaction mixture was then warmed to r.t. over 30 minutes. A solution of $\mathbf{4}$ (900 mg, $5.40 \mathrm{mmol})$ in THF $(30 \mathrm{~mL})$ was added via addition funnel to the phosphonate solution over 5 minutes. The reaction mixture was then stirred at r.t. for $18 \mathrm{~h}$. The reaction was quenched by the addition of water $(200 \mathrm{~mL})$ and then extracted with ether $(3 \times 150 \mathrm{~mL})$. The combined organic phases were dried over $\mathrm{MgSO}_{4}$, filtered, and concentrated in vacuo. The $E$ - and $Z$-isomers were separated by column chromatography ( $120 \mathrm{~g}$ silica gel cartridge, isocratic elution $10 \%$ EtOAc-hexanes); yield of 5, $0.36 \mathrm{~g}$ ( $1.8 \mathrm{mmol}, 33 \%)$ and 6, $0.23 \mathrm{~g}(1.1 \mathrm{mmol}, 17 \%)$.

$$
R_{f}=0.43 \text { (EtOAc-hexanes, } 1: 8 \text { ). }
$$

${ }^{1} \mathrm{H} \mathrm{NMR}\left(600 \mathrm{MHz}, \mathrm{CDCl}_{3}\right): \delta=6.36(\mathrm{~d}, 1 \mathrm{H}, J=12.0 \mathrm{~Hz}$, H-5), $5.96(\mathrm{dd}, 1 \mathrm{H}, J=12.0,2.0 \mathrm{~Hz}, \mathrm{H}-4), 5.50(\mathrm{~m}, 1 \mathrm{H}, \mathrm{H}-6)$, 2.85 (td, $1 \mathrm{H}, J=14.0,5.0 \mathrm{~Hz}, \mathrm{H}-9), 2.60-2.53$ (m, $1 \mathrm{H}, \mathrm{H}-9$ ), $2.09\left(\mathrm{~s}, 3 \mathrm{H}, \mathrm{O}_{2} \mathrm{CH}_{3}\right), 2.03-1.97(\mathrm{~m}, 1 \mathrm{H}, \mathrm{H}-7), 1.95(\mathrm{~s}, 3 \mathrm{H}$, $\left.\mathrm{C} 2-\mathrm{CH}_{3}\right), 1.91-1.75(\mathrm{~m}, 3 \mathrm{H}, \mathrm{H}-7, \mathrm{H}-8)$.

${ }^{13} \mathrm{C}$ NMR $\left(150 \mathrm{MHz}, \mathrm{CDCl}_{3}\right): \delta=16.3\left(\mathrm{C} 2-\mathrm{CH}_{3}\right), 21.1$ $\left(\mathrm{O}_{2} \mathrm{CCH}_{3}\right), 21.9(\mathrm{C} 8), 32.0(\mathrm{C} 7), 34.2(\mathrm{C} 9), 72.0(\mathrm{C} 6), 106.4$ (C3), 119.6 (C2), 127.1 (C5), 138.3 (C4), 151.9 (C1), 170.1 $\left(\mathrm{O}_{2} \mathrm{CCH}_{3}\right)$.

LRMS (EI): $m / z(\%)=205.1(40), 163.1(100)$. 


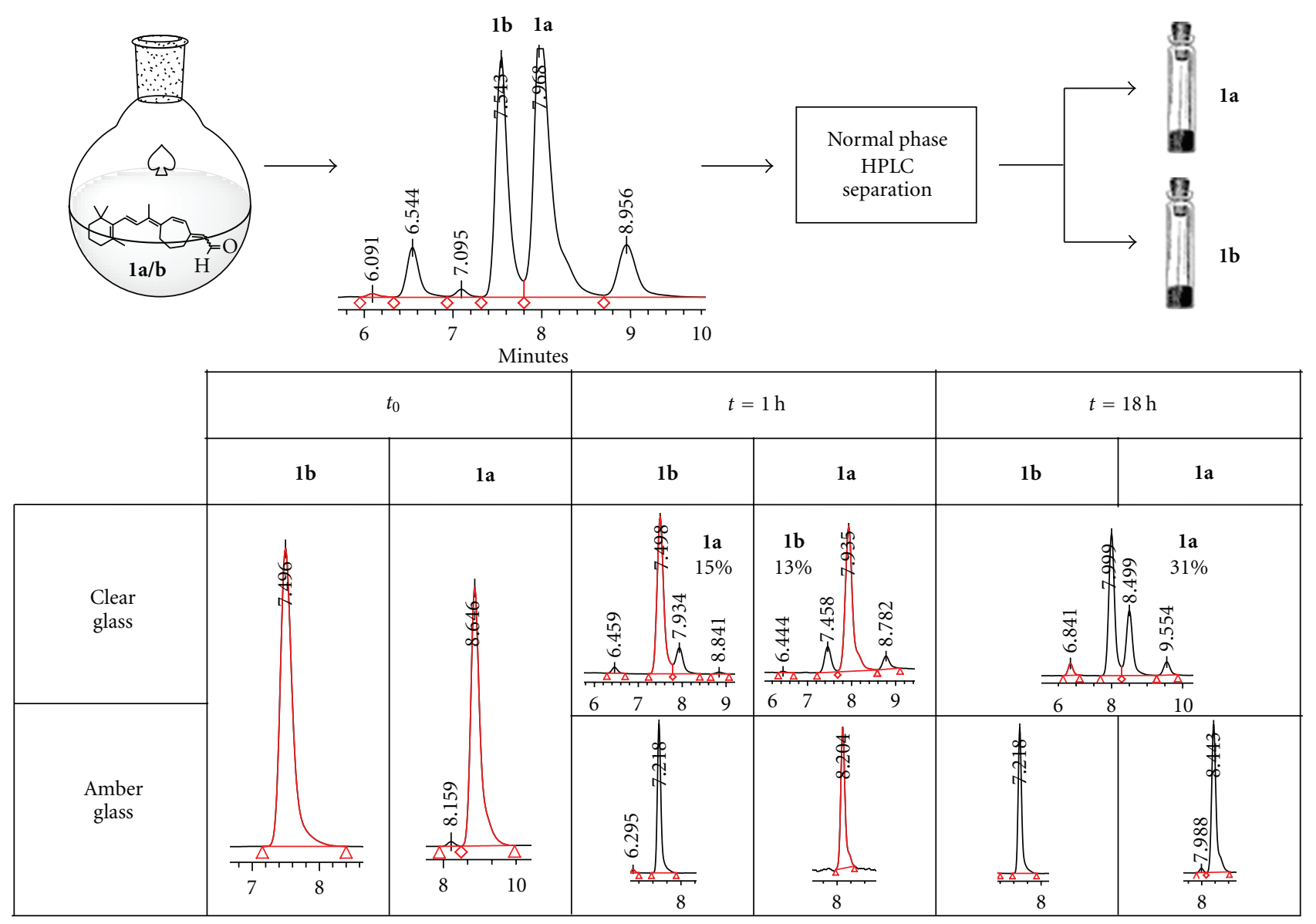

FIGURE 3: Isomerization between $\mathbf{1 a}$ and $\mathbf{1} \mathbf{b}$ under normal atmospheric conditions as monitored by HPLC (AUC at $310 \mathrm{~nm}$ ). Note that samples stored in amber glass vial show no appreciable degradation or isomerization up to $18 \mathrm{~h}$ under argon at room temperature.

(Z)-4-(1-Cyanoethylidene)cyclohept-2-en-1-yl Acetate (6).

$$
R_{f}=0.33 \text { (hexanes-EtOAc, } 8: 1 \text { ). }
$$

${ }^{1} \mathrm{H}$ NMR $\left(600 \mathrm{MHz}, \mathrm{CDCl}_{3}\right): \delta=6.64(\mathrm{~d}, 1 \mathrm{H}, J=12.0$ $\mathrm{Hz}, \mathrm{H}-5), 5.90(\mathrm{dd}, 1 \mathrm{H}, J=12.0,2.0 \mathrm{~Hz}, \mathrm{H}-4), 5.51(\mathrm{~m}, 1 \mathrm{H}$, H-6), $2.62(\mathrm{td}, 1 \mathrm{H}, J=15.0,5.0 \mathrm{~Hz}, \mathrm{H}-9), 2.40-2.31(\mathrm{~m}, 1 \mathrm{H}$, $\mathrm{H}-9), 2.07$ (s, $\left.3 \mathrm{H}, \mathrm{O}_{2} \mathrm{CH}_{3}\right), 2.04-1.98(\mathrm{~m}, 1 \mathrm{H}, \mathrm{H}-7), 1.96$ (s, $\left.3 \mathrm{H}, \mathrm{C} 2-\mathrm{CH}_{3}\right), 1.86-1.67(\mathrm{~m}, 3 \mathrm{H}, \mathrm{H}-7, \mathrm{H}-8)$.

${ }^{13} \mathrm{C} \mathrm{NMR}\left(150 \mathrm{MHz}, \mathrm{CDCl}_{3}\right): \delta=16.1\left(\mathrm{C}_{2} \mathrm{CH}_{3}\right), 20.7$ (C8), $21.1\left(\mathrm{O}_{2} \mathrm{CCH}_{3}\right), 29.8(\mathrm{C} 9), 31.9(\mathrm{C} 7), 71.7$ (C6), 106.7 (C3), 119.0 (C2), 130.0 (C5), 136.7 (C4), 152.7 (C1), 170.1 $\left(\mathrm{O}_{2} \mathrm{CCH}_{3}\right)$.

$$
\text { LRMS (EI): } m / z(\%)=205.1(15), 163.1(100) \text {. }
$$

(E)-2-(4-Hydroxycyclohept-2-en-1-ylidene)propanenitrile (7) (See [12]). To a solution of $5(320 \mathrm{mg}, 1.60 \mathrm{mmol})$ in methanol $(10 \mathrm{~mL})$ at $0^{\circ} \mathrm{C}$ was added $\mathrm{K}_{2} \mathrm{CO}_{3}(110 \mathrm{mg}$, $0.780 \mathrm{mmol}$ ). The reaction mixture was stirred for 15 minutes at $0^{\circ} \mathrm{C}$, then warmed to r.t., and stirred for $1 \mathrm{~h}$. The reaction mixture was poured into water $(25 \mathrm{~mL})$ and extracted with EtOAc $(3 \times 20 \mathrm{~mL})$. The combined organic phases were then washed with brine $(15 \mathrm{~mL})$, dried over $\mathrm{MgSO}_{4}$, filtered, and concentrated in vacuo. The product was purified by column chromatography ( $40 \mathrm{~g}$ silica gel cartridge, gradient elution 5-40\% EtOAc-hexanes); yield of 7, $0.25 \mathrm{~g}$ (1.6 mmol, 99\%).

$$
\left.R_{f}=0.11 \text { (EtOAc-hexanes, } 1: 6\right) \text {. }
$$

${ }^{1} \mathrm{H} \mathrm{NMR}\left(300 \mathrm{MHz}, \mathrm{CDCl}_{3}\right): \delta=6.26(\mathrm{~d}, 1 \mathrm{H}, J=12.0 \mathrm{~Hz}$, H-5), $6.07(\mathrm{~d}, 1 \mathrm{H}, J=12.0 \mathrm{~Hz}, \mathrm{H}-4), 4.48(\mathrm{~m}, 1 \mathrm{H}, \mathrm{H}-6), 2.79$ (td, $1 \mathrm{H}, J=10.0,5.0 \mathrm{~Hz}, \mathrm{H}-9), 2.47(\mathrm{~m}, 2 \mathrm{H}, \mathrm{H}-9, \mathrm{OH}), 2.00$ (m, $1 \mathrm{H}, \mathrm{H}-7), 1.92\left(\mathrm{~s}, 3 \mathrm{H}, \mathrm{C} 2-\mathrm{CH}_{3}\right), 1.87-1.61(\mathrm{~m}, 3 \mathrm{H}, \mathrm{H}-7$, $\mathrm{H}-8)$.

${ }^{13} \mathrm{C}$ NMR $\left(75 \mathrm{MHz}, \mathrm{CDCl}_{3}\right): \delta=16.2\left(\mathrm{C}_{2} \mathrm{CH}_{3}\right), 22.1$ (C8), 34.2 (C9), 35.6 (C7), 69.9 (C6), 105.4 (C3), 119.8 (C2), 125.4 (C5), 143.0 (C4), 152.7 (C1).

$$
\text { MS (APCI): } m / z[\mathrm{M}+\mathrm{H}]^{+}=164.1,146.1\left(-\mathrm{H}_{2} \mathrm{O}\right) .
$$

(E)-2-(4-((tert-Butyldimethylsilyl)oxy)cyclohept-2-en-1ylidene)propanenitrile (8) (See [12]). To a solution of 7 (220 mg, $1.30 \mathrm{mmol})$, imidazole $(410 \mathrm{mg}, 6.00 \mathrm{mmol})$, and DMAP $(3.00 \mathrm{mg}, 0.030 \mathrm{mmol})$ in $\mathrm{CH}_{2} \mathrm{Cl}_{2}$ at $0^{\circ} \mathrm{C}$ was added tert-butyldimethylsilyl chloride (TBSCl, $240 \mathrm{mg}$, $1.60 \mathrm{mmol}$ ). The reaction mixture was stirred at $0^{\circ} \mathrm{C}$ for 15 minutes and then warmed to r.t. for another $1.5 \mathrm{~h}$. The reaction was quenched by the addition of water $(25 \mathrm{~mL})$. 
The organic phase was removed, and the aqueous phase was extracted with $\mathrm{CH}_{2} \mathrm{Cl}_{2}(3 \times 15 \mathrm{~mL})$. The combined organic phases were washed with brine $(30 \mathrm{~mL})$, dried over $\mathrm{MgSO}_{4}$, filtered, and concentrated in vacuo. The product was purified by column chromatography $(40 \mathrm{~g}$ silica gel cartridge, gradient elution (0-5\% EtOAc-hexanes); yield of 8, $0.30 \mathrm{~g}(1.1 \mathrm{mmol}, 80 \%)$.

$$
\left.R_{f}=0.36 \text { (EtOAc-hexanes, } 1: 20\right) .
$$

${ }^{1} \mathrm{H}$ NMR $\left(600 \mathrm{MHz}, \mathrm{CDCl}_{3}\right): \delta=6.23(\mathrm{dd}, 1 \mathrm{H}, J=12.0$, $2.0 \mathrm{~Hz}, \mathrm{H}-5), 6.03(\mathrm{dd}, 1 \mathrm{H}, J=12.0,2.0 \mathrm{~Hz}, \mathrm{H}-4), 4.49-4.43$ (m, 1H, H-6), $2.82(\mathrm{td}, 1 \mathrm{H}, J=14.0,5.0 \mathrm{~Hz}, \mathrm{H}-9), 2.52$ (ddd, $1 \mathrm{H}, J=14.0,9.5,5.0 \mathrm{~Hz}, \mathrm{H}-9), 1.96-1.89(\mathrm{~m}, 4 \mathrm{H}, \mathrm{H}-7, \mathrm{C} 2-$ $\left.\mathrm{CH}_{3}\right), 1.84-1.70(\mathrm{~m}, 3 \mathrm{H}, \mathrm{H}-7, \mathrm{H}-8), 0.90\left(\mathrm{~s}, 9 \mathrm{H}, \mathrm{SiC}\left(\mathrm{CH}_{3}\right)_{3}\right)$, $0.10\left(\mathrm{~s}, 3 \mathrm{H}, \mathrm{Si}\left(\mathrm{CH}_{3}\right)_{2}\right), 0.09$ (s, $\left.3 \mathrm{H}, \mathrm{Si}\left(\mathrm{CH}_{3}\right)_{2}\right)$.

${ }^{13} \mathrm{C} \mathrm{NMR}\left(150 \mathrm{MHz}, \mathrm{CDCl}_{3}\right): \delta=-4.8\left(\mathrm{Si}\left(\mathrm{CH}_{3}\right)_{2}\right),-4.7$ $\left(\mathrm{Si}\left(\mathrm{CH}_{3}\right)_{2}\right), 16.2\left(\mathrm{C}_{2} \mathrm{CH}_{3}\right), 18.1\left(\mathrm{SiC}\left(\mathrm{CH}_{3}\right)_{3}\right), 22.2(\mathrm{C} 8), 25.8$ $\left(\mathrm{SiC}\left(\mathrm{CH}_{3}\right)_{3}\right), 34.3(\mathrm{C} 9), 35.8(\mathrm{C} 7), 70.6(\mathrm{C} 6), 105.2(\mathrm{C} 3)$, 120.0 (C2), 125.0 (C5), 144.2 (C4), 152.9 (C1).

$$
\text { MS (APCI): } m / z[\mathrm{M}+\mathrm{H}]^{+}=277.9 \text {. }
$$

(E)-2-(4-((tert-Butyldimethylsilyl)oxy)cyclohept-2-en-1ylidene)propanal (9) (See [12]). To a solution of 8 (260 mg, $0.940 \mathrm{mmol})$ in ether $(10 \mathrm{~mL})$ at $-78^{\circ} \mathrm{C}$ was added DIBAL-H ( $1 \mathrm{M}$ in $\mathrm{CH}_{2} \mathrm{Cl}_{2}, 1.41 \mathrm{~mL}, 1.40 \mathrm{mmol}$ ). The reaction mixture was stirred for 15 minutes at $-78^{\circ} \mathrm{C}$, then warmed to $0^{\circ} \mathrm{C}$, and stirred for $2 \mathrm{~h}$. The reaction was quenched by the addition of EtOAc $(3 \mathrm{~mL})$ at $0^{\circ} \mathrm{C}$. It was then poured into a vigorously stirred slurry of silica gel $(2.30 \mathrm{~g}, \sim 9$ times the mass of the nitrile) and ether $(15 \mathrm{~mL})$ under argon at r.t. The slurry was stirred for $1 \mathrm{~h}$. The silica gel was removed by vacuum filtration and rinsed with EtOAc $(100 \mathrm{~mL})$. The filtrate was concentrated in vacuo, yield of $9,0.25 \mathrm{~g}$ (0.91 mmol, 96\%).

$$
\left.R_{f}=0.25 \text { (EtOAc-hexanes, } 1: 20\right) .
$$

${ }^{1} \mathrm{H}$ NMR $\left(600 \mathrm{MHz}, \mathrm{CDCl}_{3}\right): \delta=10.2(\mathrm{~s}, 1 \mathrm{H}, \mathrm{H}-1), 6.49$ (d, $1 \mathrm{H}, J=13.0 \mathrm{~Hz}, \mathrm{H}-5), 6.08(\mathrm{~d}, 1 \mathrm{H}, J=13.0 \mathrm{~Hz}, \mathrm{H}-4), 4.48$ (m, 1H, H-6), 3.23 (td, $J=13.0,5.0 \mathrm{~Hz}, 1 \mathrm{H}, \mathrm{H}-9), 2.51-2.42$ (m, 1H, H-9), 1.95-1.88 (m, 1H, H-7), 1.86-1.74 (m, 3H), $1.80\left(\mathrm{~s}, 3 \mathrm{H}, \mathrm{C} 2-\mathrm{CH}_{3}\right), 0.93\left(\mathrm{~s}, 9 \mathrm{H}, \mathrm{SiC}\left(\mathrm{CH}_{3}\right)_{3}\right), 0.12(\mathrm{~s}, 3 \mathrm{H}$, $\left.\mathrm{Si}\left(\mathrm{CH}_{3}\right)_{2}\right), 0.11\left(\mathrm{~s}, 3 \mathrm{H}, \mathrm{Si}\left(\mathrm{CH}_{3}\right)_{2}\right)$.

${ }^{13} \mathrm{C} \mathrm{NMR}\left(150 \mathrm{MHz}, \mathrm{CDCl}_{3}\right): \delta=-4.8\left(\mathrm{Si}\left(\mathrm{CH}_{3}\right)_{2}\right),-4.7$ $\left(\mathrm{Si}\left(\mathrm{CH}_{3}\right)_{2}\right), 10.9\left(\mathrm{C}_{2} \mathrm{CH}_{3}\right), 18.1\left(\mathrm{SiC}\left(\mathrm{CH}_{3}\right)_{3}\right), 23.8(\mathrm{C} 8), 25.8$ $\left(\mathrm{SiC}\left(\mathrm{CH}_{3}\right)_{3}\right), 28.4$ (C9), 35.5 (C7), 70.5 (C6), 129.9 (C3), 133.0 (C2), 143.6 (C5), 153.9 (C4), 191.0 (C1).

$$
\text { LRMS (EI): } m / z(\%)=281.1(2) \text {. }
$$

tert-Butyldimethyl(((E)-4-((E)-4-(2,6,6-trimethyl-cyclohex1-en-1-yl)but-3-en-2-ylidene)cyclohept-2-en-1-yl)oxy)silane (11) (See $[1,12])$. To a solution of $10(4.00 \mathrm{~g}, 8.40 \mathrm{mmol})$ in $\mathrm{CH}_{2} \mathrm{Cl}_{2}(30 \mathrm{~mL})$ at r.t. in the dark was added potassium tertbutoxide $(940 \mathrm{mg}, 8.40 \mathrm{mmol})$ and 18 -crown-6 $(55.0 \mathrm{mg}$, $0.210 \mathrm{mmol}$ ). The solution was stirred for 15 minutes at r.t., then a solution of $9(1.20 \mathrm{~g}, 4.20 \mathrm{mmol})$ in $\mathrm{CH}_{2} \mathrm{Cl}_{2}$ was added. The reaction mixture was stirred in the dark for $5 \mathrm{~h}$ at r.t. The reaction was quenched by the addition of water $(100 \mathrm{~mL})$ and extracted with ether $(3 \times 100 \mathrm{~mL})$. The combined organic layers were washed with brine $(100 \mathrm{~mL})$, dried over $\mathrm{Na}_{2} \mathrm{SO}_{4}$, filtered, and concentrated in vacuo. The product was purified by column chromatography $(330 \mathrm{~g}$ silica gel cartridge, gradient elution 0-1\% EtOAc-hexanes); yield of $11,0.59 \mathrm{~g}(1.5 \mathrm{mmol}, 35 \%)$.

$$
\left.R_{f}=0.9 \text { (EtOAc-hexanes, } 1: 10\right) .
$$

${ }^{1} \mathrm{H}$ NMR $\left(400 \mathrm{MHz}, \mathrm{CDCl}_{3}\right): \delta=6.52(\mathrm{~d}, 1 \mathrm{H}, J=$ $16.0 \mathrm{~Hz}), 6.49(\mathrm{dd}, 1 \mathrm{H}, J=12.0,2.0 \mathrm{~Hz}), 6.20(\mathrm{~d}, 1 \mathrm{H}, J=$ $16.0 \mathrm{~Hz}), 5.70(\mathrm{dd}, 1 \mathrm{H}, J=12.0,2.0 \mathrm{~Hz}), 4.53-4.46(\mathrm{~m}, 1 \mathrm{H})$, $2.71(\mathrm{td}, 1 \mathrm{H}, J=13.5,5.0 \mathrm{~Hz}), 2.31-2.20(\mathrm{~m}, 1 \mathrm{H}), 2.10-1.94$ $(\mathrm{m}, 2 \mathrm{H}), 1.90(\mathrm{~s}, 3 \mathrm{H}), 1.87-1.76(\mathrm{~m}, 2 \mathrm{H}), 1.74(\mathrm{~s}, 3 \mathrm{H}), 1.72-$ $1.54(\mathrm{~m}, 4 \mathrm{H}), 1.47(\mathrm{~m}, 2 \mathrm{H}), 1.06(\mathrm{~s}, 3 \mathrm{H}), 1.05(\mathrm{~s}, 3 \mathrm{H}) 0.92(\mathrm{~s}$, $9 \mathrm{H}), 0.12(\mathrm{~s}, 3 \mathrm{H}), 0.11(\mathrm{~s}, 3 \mathrm{H})$.

$$
\text { MS (ES): } m / z[\mathrm{M}+\mathrm{H}]^{+}=400.1 \text {. }
$$

(E)-4-((E)-4-(2,6,6-Trimethylcyclohex-1-en-1-yl)but-3-en2-ylidene)cyclohept-2-enone (12) (See $[1,7,12])$. To a solution of $11(390 \mathrm{mg}, 0.960 \mathrm{mmol})$ in THF $(32 \mathrm{~mL})$ in the dark, at $0^{\circ} \mathrm{C}$, was added TBAF $(1 \mathrm{M}$ in THF, $1.90 \mathrm{~mL}$, $1.92 \mathrm{mmol}$ ). The reaction mixture was stirred in the dark at $0^{\circ} \mathrm{C}$ for $5 \mathrm{~h}$. The reaction was quenched by the addition of water $(35 \mathrm{~mL})$ and extracted with EtOAc $(3 \times 35 \mathrm{~mL})$. The combined organic layers were washed with brine $(50 \mathrm{~mL})$, dried over $\mathrm{Na}_{2} \mathrm{SO}_{4}$, filtered, and concentrated in vacuo. The allylic alcohol $\left(540 \mathrm{mg}\right.$, yellow solid, $R_{f}=0.24$ (hexanes-EtOAc 10:1)) was dissolved in $\mathrm{CH}_{2} \mathrm{Cl}_{2}(15 \mathrm{~mL})$ and cooled to $-20^{\circ} \mathrm{C}$ in the dark. $\mathrm{MnO}_{2}(85 \%$ activated, $1.70 \mathrm{~g}, 20.0 \mathrm{mmol}$ ) was added, and the mixture was stirred for 10 minutes in the dark at $-20^{\circ} \mathrm{C}$. The reaction mixture was then warmed to r.t. and stirred for $2 \mathrm{~h}$ in the dark. The reaction mixture was then vacuum filtered through a pad of Celite, and the cake was washed with $\mathrm{CH}_{2} \mathrm{Cl}_{2}(5 \times 10 \mathrm{~mL})$. The bright yellow filtrate was concentrated in vacuo. The product was purified by column chromatography ( $40 \mathrm{~g}$ silica gel cartridge, gradient elution 0-20\% EtOAc-hexanes); yield of $12,0.13 \mathrm{~g}(0.45 \mathrm{mmol}, 47 \%)$.

$$
\left.R_{f}=0.18 \text { (EtOAc-hexanes, } 1: 20\right) .
$$

${ }^{1} \mathrm{H} \mathrm{NMR}\left(400 \mathrm{MHz}, \mathrm{CDCl}_{3}\right): \delta \mathrm{ppm} 7.39(\mathrm{~d}, 1 \mathrm{H}, J=12.0$ $\mathrm{Hz}), 6.61(\mathrm{~d}, 1 \mathrm{H}, J=16.0 \mathrm{~Hz}), 6.45(\mathrm{~d}, 1 \mathrm{H}, J=16.0 \mathrm{~Hz}), 5.96$ $(\mathrm{d}, 1 \mathrm{H}, J=12.0 \mathrm{~Hz}), 2.65(\mathrm{t}, 2 \mathrm{H}, J=6.5 \mathrm{~Hz}), 2.62(\mathrm{t}, 2 \mathrm{H}, J=$ $6.5 \mathrm{~Hz}), 2.06(\mathrm{~m}, 5 \mathrm{H}), 1.96-1.87(\mathrm{~m}, 2 \mathrm{H}), 1.76(\mathrm{~s}, 3 \mathrm{H}), 1.70-$ $1.62(\mathrm{~m}, 2 \mathrm{H}), 1.57(\mathrm{~s}, 1 \mathrm{H}), 1.51(\mathrm{~m}, 2 \mathrm{H}), 1.07(\mathrm{~s}, 6 \mathrm{H})$.

$$
\text { MS (ES): } m / z[\mathrm{M}+\mathrm{H}]^{+}=285.1 \text {. }
$$

(E)-2-((E)-4-((E)-4-(2,6,6-trimethylcyclohex-1-en-1-yl)but3-en-2-ylidene)cyclohept-2-en-1-ylidene) Acetaldehyde (1a) (See [12]). Into a dried round bottom flask containing $\mathrm{NaH}$ (60\% dispersion in oil, $151 \mathrm{mg}, 4.50 \mathrm{mmol}$ ) and THF (7 mL) was added diethyl cyanomethylphosphonate $(0.710 \mathrm{~mL}$, $4.50 \mathrm{mmol}$ ) over 15 minutes. The solution was stirred for 20 
minutes at r.t., then the flask was wrapped in aluminum foil. A solution of the $12(130 \mathrm{mg}, 0.450 \mathrm{mmol})$ in THF $(6 \mathrm{~mL})$ was added to the reaction mixture, and the reaction was stirred for $3 \mathrm{~h}$ at r.t. in the dark. The reaction was poured into $30 \mathrm{~mL}$ of ice water and then extracted with ether $(4 \times$ $20 \mathrm{~mL}$ ). The combined organic phases were washed with brine $(20 \mathrm{~mL})$, dried over $\mathrm{MgSO}_{4}$, filtered, and concentrated in vacuo. The intermediate nitrile was purified by column chromatography ( $40 \mathrm{~g}$ silica gel cartridge, isocratic 5\% EtOAc-hexane); yield of pentaene nitrile $0.14 \mathrm{~g}(0.45 \mathrm{mmol}$, $99 \%)$.

$$
R_{f}=0.36(\text { EtOAc-hexanes, } 1: 50) .
$$

To a solution of the nitrile ( $140 \mathrm{mg}, 0.450 \mathrm{mmol}$ ) in $\mathrm{Et}_{2} \mathrm{O}$ $(60 \mathrm{~mL})$ at $-78^{\circ} \mathrm{C}$ in the dark was added DIBAL-H $(1 \mathrm{M}$ in $\left.\mathrm{CH}_{2} \mathrm{Cl}_{2}, 3.00 \mathrm{~mL}, 3.00 \mathrm{mmol}\right)$. The reaction mixture was stirred at $-78^{\circ} \mathrm{C}$ for 10 minutes, then warmed to r.t., and stirred for 30 minutes in the dark. It was then poured into a vigorously stirred slurry of silica gel $(5.50 \mathrm{~g}, \sim 40$ times the mass of the nitrile) and ether $(20 \mathrm{~mL})$ under argon at r.t. The slurry was stirred for 30 minutes at r.t. The silica gel was removed by filtration under argon, and the silica gel was washed with ether $(100 \mathrm{~mL})$. The bright yellow filtrate was concentrated in vacuo, yield of $E / Z-10.14 \mathrm{~g}(0.45 \mathrm{mmol}$, $96 \%, E: Z$ ratio $\sim 1: 1)$. The product was purified by HPLC using a normal phase semiprep column (Phenomenex Luna $5 \mu$ silica $100 \AA, 250 \mathrm{~mm} \times 10 \mathrm{~mm} \times 5 \mathrm{~mm})$, isocratic elution $5 \%$ ether-hexanes, and flow rate of $10 \mathrm{~mL} \mathrm{~min}^{-1}$ and monitored at 350 and $210 \mathrm{~nm}$. For optimal separation of isomers $1 \mathrm{mg}$ aldehyde in $0.2 \mathrm{~mL}$ hexanes (HPLC grade) was injected per run. This provided 1a with an isomeric purity of $96 \%$ by HPLC (AUC at $350 \mathrm{~nm}$ ), and $\mathbf{1 b}$ with an isomeric purity of $>98 \%$ by HPLC (AUC at $350 \mathrm{~nm}$ ) (see Figure 3, panels $\mathbf{1} \mathbf{a}$ and $\mathbf{1} \mathbf{b}$ at $\left.t_{0}\right)$.

$$
\left.R_{t}=8.13 \mathrm{~min} ; R_{f}=0.35 \text { (EtOAc-hexanes, } 1: 20\right) .
$$

${ }^{1} \mathrm{H} \mathrm{NMR}\left(400 \mathrm{MHz} \mathrm{CDCl}_{3}\right): \delta=10.07(\mathrm{~d}, 1 \mathrm{H}, J=8.0 \mathrm{~Hz}$, $\mathrm{H}-15), 6.95$ (d, $1 \mathrm{H}, J=11.5 \mathrm{~Hz}, \mathrm{H}-11), 6.57$ (d, $1 \mathrm{H}, J=$ $16.0 \mathrm{~Hz}, \mathrm{H}-8), 6.38$ (d, 1H, J = 16.0 Hz, H-7), 6.26 (d, 1H, J $=11.5 \mathrm{~Hz}, \mathrm{H}-12), 5.97(\mathrm{~d}, 1 \mathrm{H}, J=8.0 \mathrm{~Hz}, \mathrm{H}-14), 2.89$ (t, $2 \mathrm{H}$, $J=6.5 \mathrm{~Hz}, \mathrm{H}-21), 2.60(\mathrm{t}, 2 \mathrm{H}, J=6.5 \mathrm{~Hz}, \mathrm{H}-20), 2.12-2.00$ $\left(\mathrm{m}, 3 \mathrm{H}, \mathrm{C} 9-\mathrm{CH}_{3}\right), 1.94(\mathrm{~m}, 2 \mathrm{H}, \mathrm{H}-4), 1.76\left(\mathrm{~s}, 3 \mathrm{H}, \mathrm{C} 5-\mathrm{CH}_{3}\right)$, $1.71-1.60$ (m, 2H, H-3), 1.51 (m, 2H, H-2), 1.13-1.03 (s, 6H, $\left.2 \mathrm{xC} 1-\mathrm{CH}_{3}\right)$.

$$
\text { MS (ES): } m / z[\mathrm{M}+\mathrm{H}]^{+}=311.0 \text {. }
$$

(Z)-2-((E)-4-((E)-4-(2,6,6-trimethylcyclohex-1-en-1-yl) but3-en-2-ylidene)cyclohept-2-en-1-ylidene) Acetaldehyde (1b).

$$
\left.R_{t}=7.69 \mathrm{~min} ; R_{f}=0.35 \text { (EtOAc-hexanes, } 1: 20\right) \text {. }
$$

${ }^{1} \mathrm{H}$ NMR (400 MHz, $\mathrm{CD}_{2} \mathrm{Cl}_{2}$ ): $\delta$ ppm $10.15(\mathrm{~d}, 1 \mathrm{H}, J=$ $8.0 \mathrm{~Hz}, \mathrm{H}-15), 7.07$ (m, 2H, H-11, H-12), 6.61 (d, 1H, J = $15.5 \mathrm{~Hz}, \mathrm{H}-8$ ), 6.40 (d, 1H, J = $15.5 \mathrm{~Hz}, \mathrm{H}-7), 5.80$ (d, 1H, J $=8.0 \mathrm{~Hz}, \mathrm{H}-14), 2.57(\mathrm{t}, 2 \mathrm{H}, J=7.0 \mathrm{~Hz}, \mathrm{H}-21), 2.47(\mathrm{t}, 2 \mathrm{H}, J$ $=7.0 \mathrm{~Hz}, \mathrm{H}-20), 2.03\left(\mathrm{~m}, 5 \mathrm{H}, \mathrm{H}-4, \mathrm{C} 9-\mathrm{CH}_{3}\right), 1.95-1.85(\mathrm{~m}$, $2 \mathrm{H}, \mathrm{H}-21), 1.76\left(\mathrm{~s}, 3 \mathrm{H}, \mathrm{C} 5-\mathrm{CH}_{3}\right), 1.72-1.62$ (m, 2H, H-3), $1.30(\mathrm{~m}, 2 \mathrm{H}, \mathrm{H}-2), 1.07$ (s, 6H, 2xC1- $\left.\mathrm{CH}_{3}\right)$.

$$
\text { MS (ES): } m / z[\mathrm{M}+\mathrm{H}]^{+}=311.0 \text {. }
$$

\section{Acknowledgments}

The autors thank Dr. Howard Hunter of York University (Toronto, ON) for NMR experiments. They also thank Dr. Muz Mansuri of Bikam Pharmaceuticals for his helpful advice and discussion during the preparation of this paper.

\section{References}

[1] S. M. Noorwez, V. Kuksa, Y. Imanishi et al., "Pharmacological chaperone-mediated in vivo folding and stabilizationof the $\mathrm{P} 23 \mathrm{H}$-opsin mutant associated with autosomal dominant retinitis pigmentosa," Journal of Biological Chemistry, vol. 278, no. 16, pp. 14442-14450, 2003.

[2] S. M. Noorwez, R. Malhotra, J. H. McDowell, K. A. Smith, M. P. Krebs, and S. Kaushal, "Retinoids assist the cellular folding of the autosomal dominant retinitis pigmentosa opsin mutant P23H," Journal of Biological Chemistry, vol. 279, no. 16, pp. 16278-16284, 2004.

[3] V. Kuksa, F. Bartl, T. Maeda et al., "Biochemical and physiological properties of rhodopsin regenerated with 11-cis-6-ringand 7-ring-retinals," Journal of Biological Chemistry, vol. 277, no. 44, pp. 42315-42324, 2002.

[4] T. Zankel, H. Ok, R. Johnson et al., "Bovine rhodopsin with 11-cis-locked retinal chromophore neither activates rhodopsin kinase nor undergoes conformational change upon irradiation," Journal of the American Chemical Society, vol. 112, no. 13, pp. 5387-5388, 1990.

[5] D. W. Corson, M. C. Cornwall, E. F. MacNichol et al., "Sensitization of bleached rod photoreceptors by 11-cis-locked analogues of retinal," Proceedings of the National Academy of Sciences of the United States of America, vol. 87, no. 17, pp. 6823-6827, 1990.

[6] J. Lou, Q. Tan, E. Karnaukhova, N. Berova, K. Nakanishi, and R. K. Crouch, "Synthetic retinals: convenient probes of rhodopsin and visual transduction process," in Methods in Enzymology, vol. 315, pp. 219-237, Elsevier, 2000.

[7] H. Akita, S. P. Tanis, M. Adams, V. Balogh-Nair, and K. Nakanishi, "Nonbleachable rhodopsins retaining the full natural chromophore," Journal of the American Chemical Society, vol. 102, no. 20, pp. 6370-6372, 1980.

[8] C. G. Caldwell, F. Derguini, C. F. Bigge et al., "Synthesis of retinals with eight- and nine-membered rings in the side chain. Models for rhodopsin photobleaching intermediates," Journal of Organic Chemistry, vol. 58, no. 13, pp. 3533-3537, 1993.

[9] E. J. Corey, D. Enders, and M. G. Bock, "A simple and highly effective route to $\alpha, \beta$-unsaturated aldehydes," Tetrahedron Letters, vol. 17, no. 1, pp. 7-10, 1976.

[10] O. Isler, H. Gutmann, H. Lindlar et al., "Synthesen in der Carotinoid-Reihe. 6. Synthese von crocetindialdhyd und lycopin," Helvetica Chimica Acta, vol. 39, pp. 463-473, 1956.

[11] M. I. Dawson, P. D. Hobbs, R. L. S. Chan, and W. R. Chao, "Retinoic acid analogues with ring modifications.Synthesis and pharmacological activity," Journal of Medicinal Chemistry, vol. 24, no. 10, pp. 1214-1223, 1981.

[12] Y. Fujimoto, R. Xie, S. E. Tully, N. Berova, and K. Nakanishi, "Synthesis of 11-cis-locked bicyclo[5.1.0] octanyl retinal and an enantioselective binding to bovine opsin," Chirality, vol. 14, no. 4, pp. 340-346, 2002, Scheme 1 lists $n$-BuLi as the base whereas the experimental lists $\mathrm{KO} t \mathrm{Bu}$ and 18-crown-6; this discrepancy led us to test both conditions. 
[13] A. Hudson, M. A. D. Bona, D. Waterman, A. Alberti, M. Benaglia, and D. Macciantelli, "Free radicals from cyclic enones: an electron paramagnetic resonance investigation. Part 1. Radicals formed by hydrogen abstraction," Journal of the Chemical Society. Perkin Transactions 2, no. 12, pp. 24872490, 1997. 


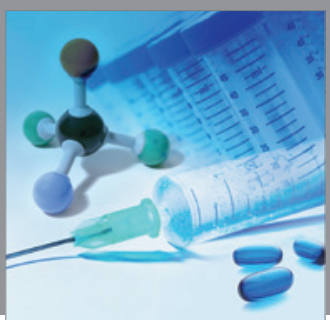

International Journal of

Medicinal Chemistry

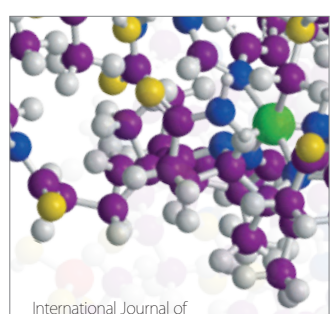

Carbohydrate Chemistry

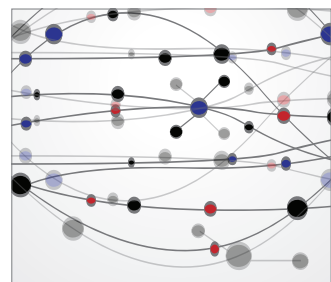

The Scientific World Journal
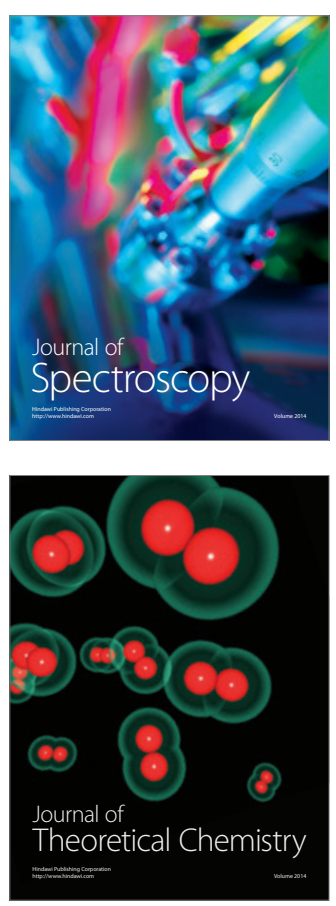
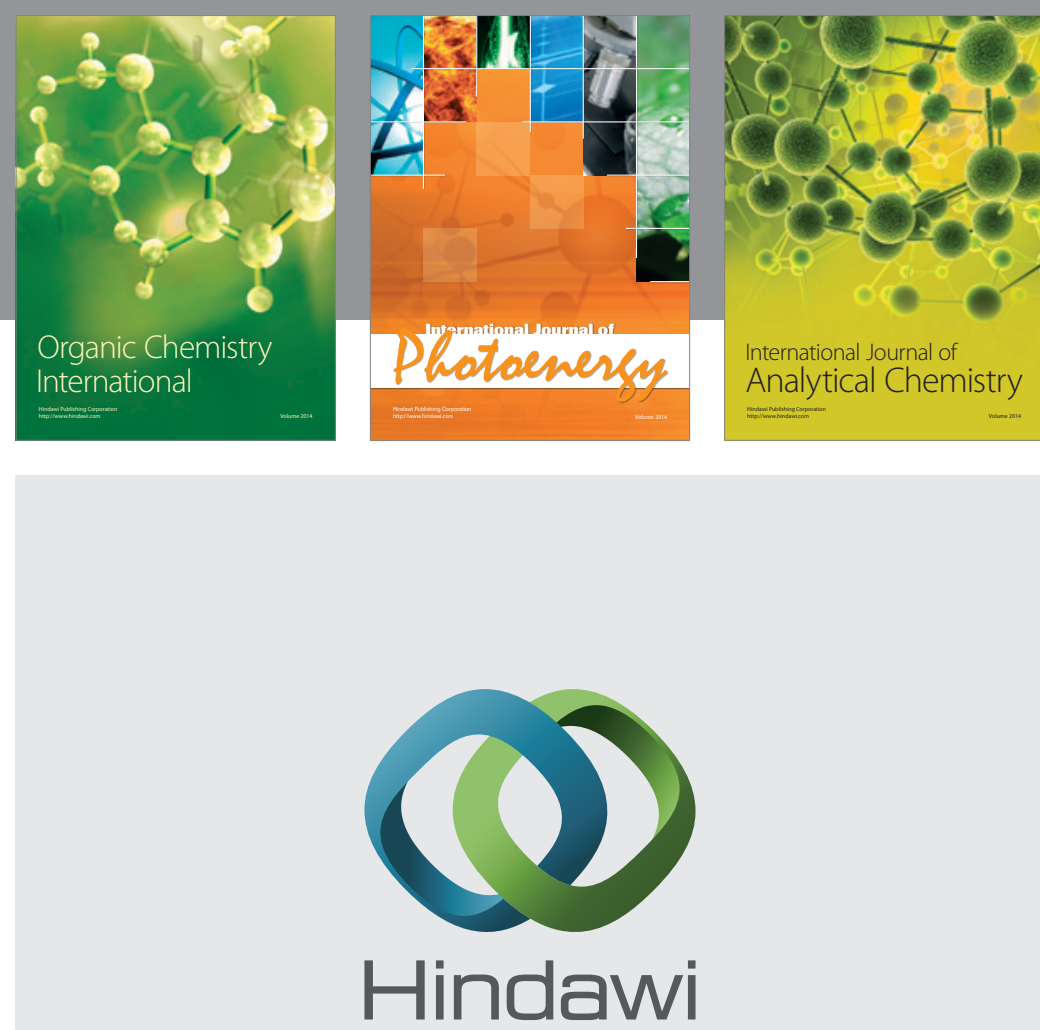

Submit your manuscripts at

http://www.hindawi.com
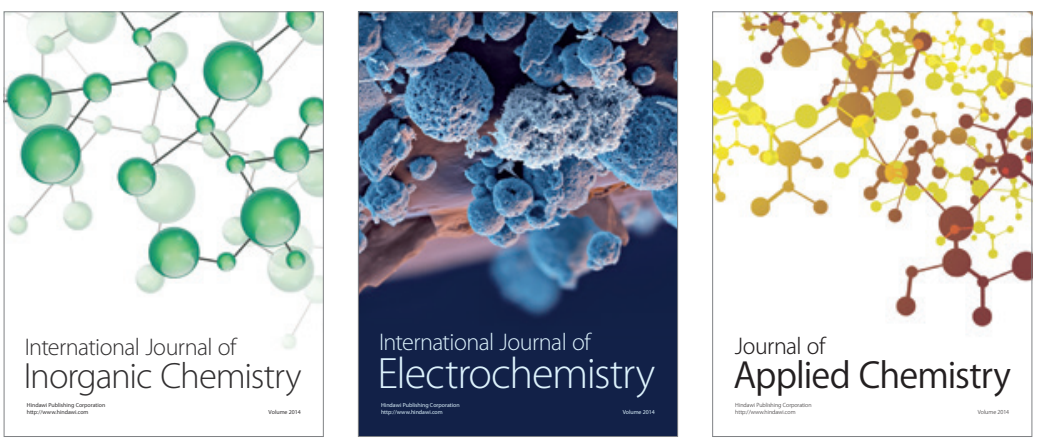

Journal of

Applied Chemistry
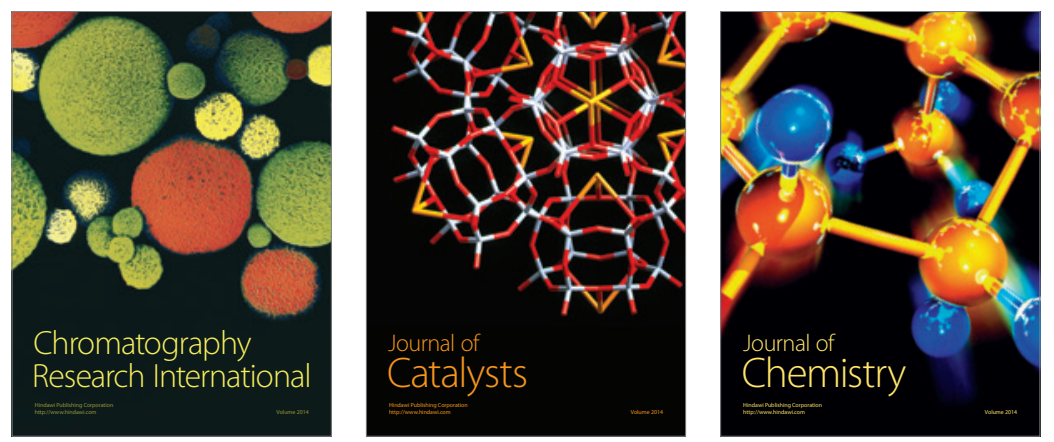
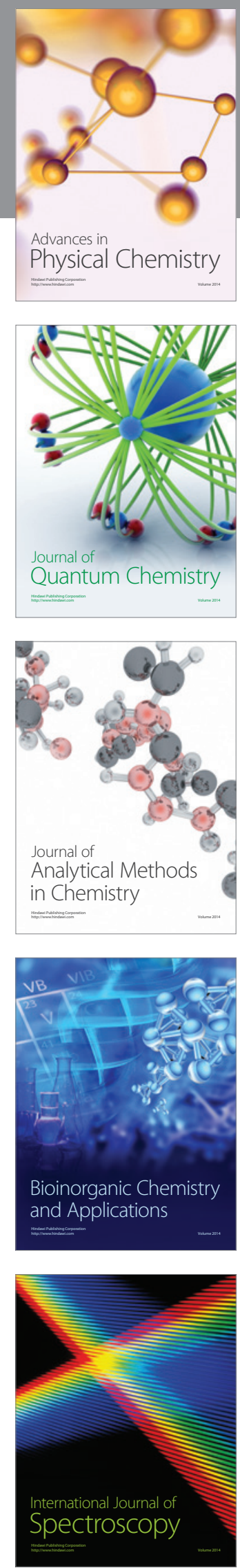